\title{
Percutaneous implantation of pedicle screws via a different trajectory: a minimally invasive surgical option for screw loosening in osteopenic or osteoporotic conditions
}

\author{
Kyoung Hwan Yoo, Pius Kim, Chang Il Ju, Seok Won Kim \\ Department of Neurosurgery, Chosun University College of Medicine, Gwangju, Korea
}

Objective: The aim of this study was to evaluate the feasibility and safety of screw reimplantation via a different trajectory as a minimally invasive alternative to extensive open surgery for symptomatic low-grade screw loosening.

Methods: Among patients who underwent single-level posterior lumbar interbody fusion and percutaneous screw fixation, a consecutive series of 10 patients in whom percutaneous screw reimplantation was performed via a different trajectory due to pedicle screw loosening were included in this study. Pedicle screw loosening was deemed clinically relevant in cases of continuous back pain with radiolucent halo zones at a vertebral level. All patients were treated via a posterior approach with removal of the loosened screws and augmentation of the allograft bone chips, followed by reimplantation with a different downward and medial trajectory using a larger-diameter screw. Demographic, preoperative, and postoperative data were collected and analyzed.

Results: The loosened screws could be removed without difficulty, and reimplantation was successfully performed via a new trajectory with larger screws in all patients. All patients reported significant pain relief at the final follow-up. Moreover, no severe complications, such as wound infection or repeated screw loosening, were observed during the follow-up period.

Conclusion: Percutaneous reimplantation of screws via a different trajectory for pedicle screw loosening with larger screws can be safely attempted in selected patients to relieve pain and avoid extensive long-level screw fixation.

Keywords: Pedicle; Loosening; Trajectory

\section{Introduction}

Posterior screwing with interbody fusion is commonly performed for the treatment of many different spine pathologies. Despite immediate and strong fixation, as well as a relatively few device-related complications, screw fixation for the purpose of bone fusion in the soteopenic or osteoporotic spine still remains a challenge [1,2]. A typical complication of pedicle screw fixation is screw loosening and the estimated rate ranges from $0.8 \%$ to $27 \%$, and the incidence rate increases rapidly in cases of severe osteoporotic spine [3,4].

In cases of symptomatic pedicle screw loosening unfortunately,

Received: February 3, $2021 \quad$ Accepted: February 15, 2021

Corresponding Author: Seok Won Kim, MD

Department of Neurosurgery, Chosun University College of Medicine, 365

Pilmun-daero, Dong-gu, Gwangju 61453, Korea

E-mail:chosunns@chosun.ac.kr few treatment options exist. The typical treatment is extensive open surgery with hard ware revision such as extended fusion level and multisegmental screw fixation.

Recently, due to the reluctance of the surgeon for extensive revision surgery, minimally invasive cement augmentation using polymethylmethacrylate (PMMA) to treat cases of clinically relevant pedicle screw loosening has been reported $[5,6]$. However, as far as the authors' knowledge, cement augmentation has not been proven as the definitive method of alleviating pain resulting from screw loosening. Moreover, screw loosening because of bone resorption around implants can be caused by spinal infections, which can be a contraindication for PMMA augmentation [7].

The aim of this study was to assess the results of percutaneous reimplantation of pedicle screws via a different trajectory for clinically relevant screw loosening, which is usually requires extensive revision surgery with multisegmental screw fixation. 


\section{Material and Method}

This study was approved by the Institutional Review Bord of Chosun University Hospital (IRB No: 2020-01-007-006) which waved the requirement for informed consent due to the respective nature of study.

We obtained and analyzed the records of 10 consecutive patients with symptomatic pedicle screw loosening who had undergone percutaneous reimplantation of screws from June 2015 to July 2018.

These patients had undergone previous single-level lumbar interbody fusion and percutaneous screw fixation for degenerative lumbar spine diseases, complicated by symptomatic pedicle screw loosening. The surgical inclusion criteria were as follows: (1) development of continuous para-midline back pain resistant to conservative treatments after posterior lumbar interbody fusion (PLIF) surgery; (2) typical finding of radiolucent halo zones around the screws at a vertebral level on both plain radiography and computed tomography (CT); and (3) screw loosening without a prominent screw back-out or strip which needed extensive open surgery. Patients with significant coagulopathy or prominent spondylitis with marked level of elevated infection markers at the time at previous lumbar interbody fusion were excluded.

Demographic data of the 10 patients were obtained and analyzed for their age, sex, preoperative diagnosis, bone mineral density, and modified MacNab's criteria at final follow-up (Table 1). The patients underwent percutaneous reimplantation of screws via a different trajectory with larger-diameter screws. Follow-up was performed clinically and radiologically 1, 2, 3, 6 months after surgery and at final follow-up with a clinical visit and using standing plain radiographic films.

\section{Surgical technique}

Under general anesthesia, the patient was placed in the prone posi- tion. A paraspinal longitudinal skin incision was made at the site of previous percutaneous screw fixation. Subsequently, the wound was dissected, and the loosened screws and rods were removed (Fig. 1A), most of which did not require much effort. Soft tissues in the previous screw fixation site were dissected, and massive irrigation was performed. The tract was palpated with a straight probe to identify the pedicle wall; allograft bone chips were used to fill the tract of

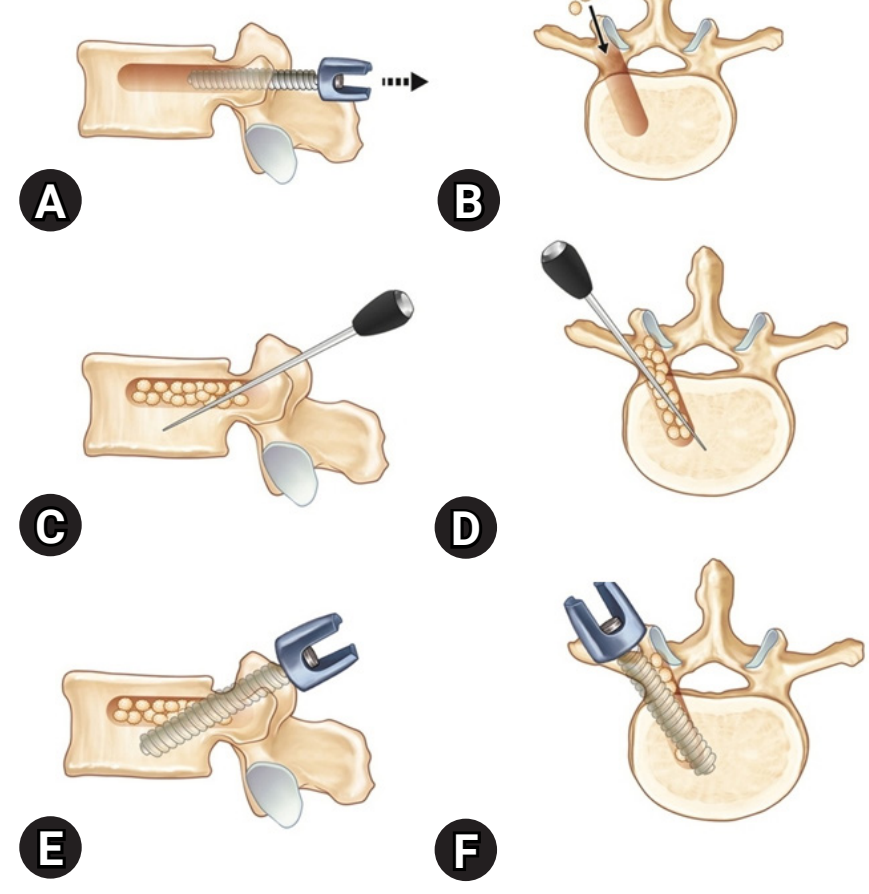

Fig. 1. Schematic illustrations of percutaneous reimplantation via a different trajectory for pedicle screw loosening. (A) The previously loosened screw is removed. (B) Allograft bone chips are packed into the space from which the previous screw was removed. (C, D) A new trajectory is targeted from a relatively lateral direction to the medial and cephalad directions and finally to the caudal direction. $(E, F) A$ longer, thicker permanent screw is inserted.

Table 1. Demographic characteristics of the patients

\begin{tabular}{cccccccc}
\hline Case & Age (yr) /sex & Level & BMD (mean) & Time until revision surgery (mo) & Follow-up (mo) & Remarks & Modified MacNab's criteria \\
\hline 1 & $68 / \mathrm{M}$ & L4-5 & -2.9 & 5 & 12 & Local infection & $\mathrm{E}$ \\
2 & $66 / \mathrm{M}$ & L4-5 & -3.0 & 10 & 14 & Local infection & $\mathrm{E}$ \\
3 & $73 / \mathrm{F}$ & L4-5 & -2.4 & 17 & 10 & - & $\mathrm{G}$ \\
4 & $58 / \mathrm{M}$ & L4-5 & -2.4 & 9 & 10 & - & $\mathrm{E}$ \\
5 & $72 / \mathrm{M}$ & L4-5 & -3.2 & 8 & 11 & - & $\mathrm{E}$ \\
6 & $60 / \mathrm{F}$ & L3-4 & -3.9 & 10 & 12 & - & $\mathrm{E}$ \\
7 & $62 / \mathrm{M}$ & L4-5 & -4.0 & 8 & 9 & - & $\mathrm{E}$ \\
8 & $76 / \mathrm{M}$ & L4-5 & -3.0 & 11 & 12 & - & $\mathrm{F}$ \\
9 & $59 / \mathrm{F}$ & L4-5 & -2.0 & 13 & 12 & - & $\mathrm{G}$ \\
10 & $67 / \mathrm{M}$ & L5-S1 & -2.9 & 8 & 10 & $\mathrm{DM}, \mathrm{H} / \mathrm{T}$ & $\mathrm{E}$
\end{tabular}

$B M D$, bone mineral densitometry; $E$, excellent; $G$, good; $F$, fair; $D M$, diabetes mellitus; $H / T$, hypertension. 
the removed screw to the maximum possible level (Fig. 1B).

The new screw entry point was generally superior to the previous screw insertion site. The new trajectory was targeted to pass over the anterior three quarters of the vertebral body to the midline, crossing the course of the loosened screw at the level of the posterior wall and running obliquely from the lateral direction to the medial and cephalad directions and finally to the caudal direction (Fig. 1C, D). After guiding the new transpedicular screw to the new entry point, it was inserted following the new trajectory (Fig. 1E, F). If there was no evidence of screw loosening, allograft bone chips were used to fill the tract of the removed screw to the maximum possible level, and a new same-size screw was inserted following the previous trajectory. After we confirmed that the new screw, which had a larger diameter compared to the previous screw, was successfully inserted in place, the operative wound was closed in layers with Vicryl sutures using an auto-stapler (Figs. 2 and 3).

\section{Safety and outcome measurement}

Patients were evaluated during the follow-up period using the visu- al analogue scale (VAS) and with the modified MacNab's criteria for characterizing clinical outcomes of spinal surgery at the final follow-up. The paired t-test was used for comparisons at different time points, and differences were considered statistically significant at $\mathrm{P}<0.05$.

\section{Results}

A total of 10 patients ( 7 males and 3 females) underwent percutaneous reimplantation of larger screws via a different trajectory. All patients had newly developed back pain after a period of pain relief, and the mean revision time was 9.9 months from previous PLIF surgery.

The mean age of the patients was 66.1 years (range, 58-76 years), and the mean follow-up period was 11.2 months (range, 6-14 months). Table 1 presents the demographics of all study participants. All patients had osteopenia or osteoporosis. Screws could be inserted via a new trajectory in all patients.

At the final follow-up after the revision surgery, nine patients
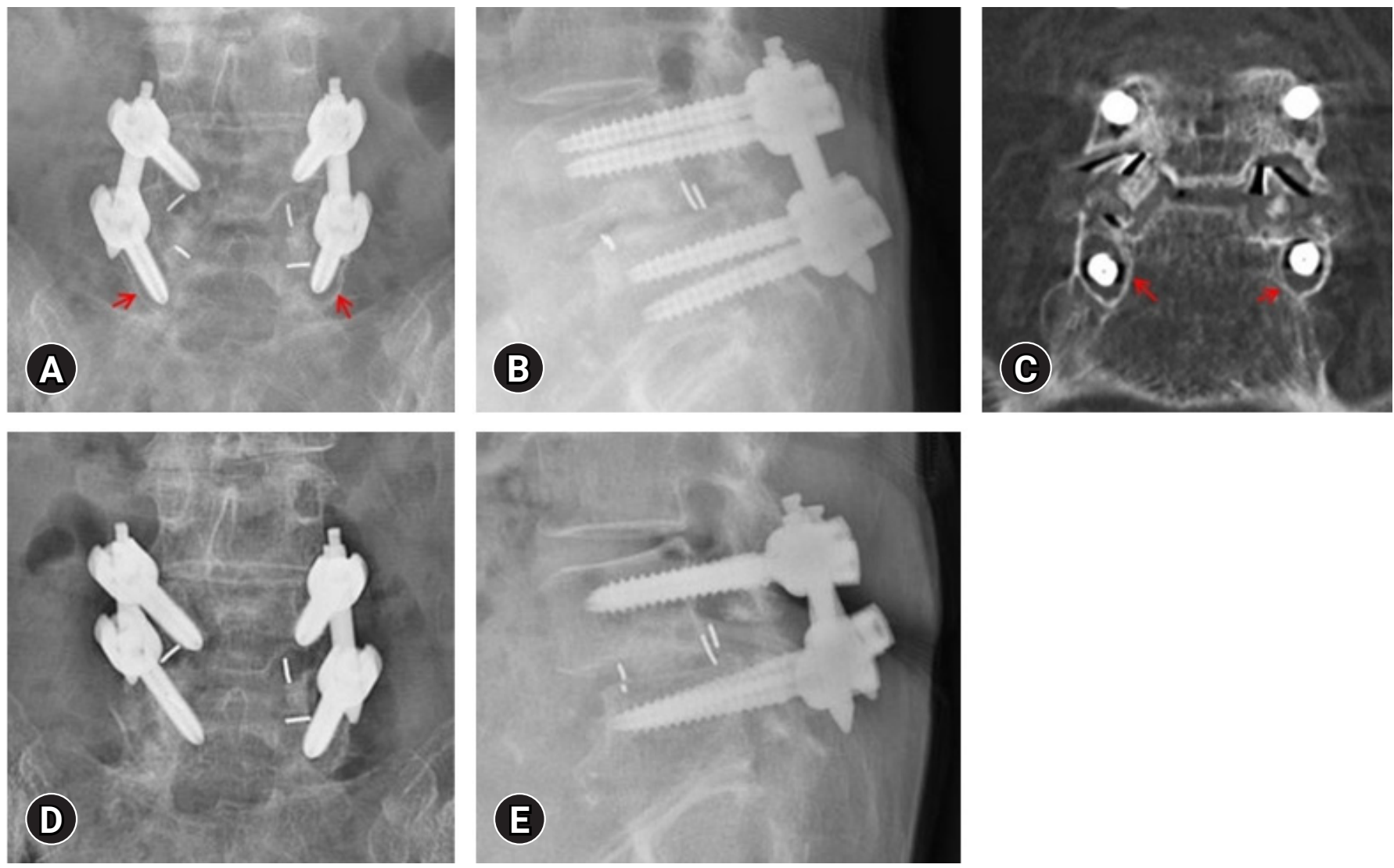

Fig. 2. A 73-year-old female who underwent L4-5 lumbar interbody fusion 17 months earlier. (A, B) Simple anteroposterior and lateral radiographs show radiolucent halos around both $\mathrm{L} 5$ pedicle screws (arrows). (C) Coronal computed tomography reveals loosening of both L5 screws (arrows). (D, E) Postoperative simple anteroposterior and lateral radiographs obtained 6 months after revision surgery show adequate repositioning of the screws. 

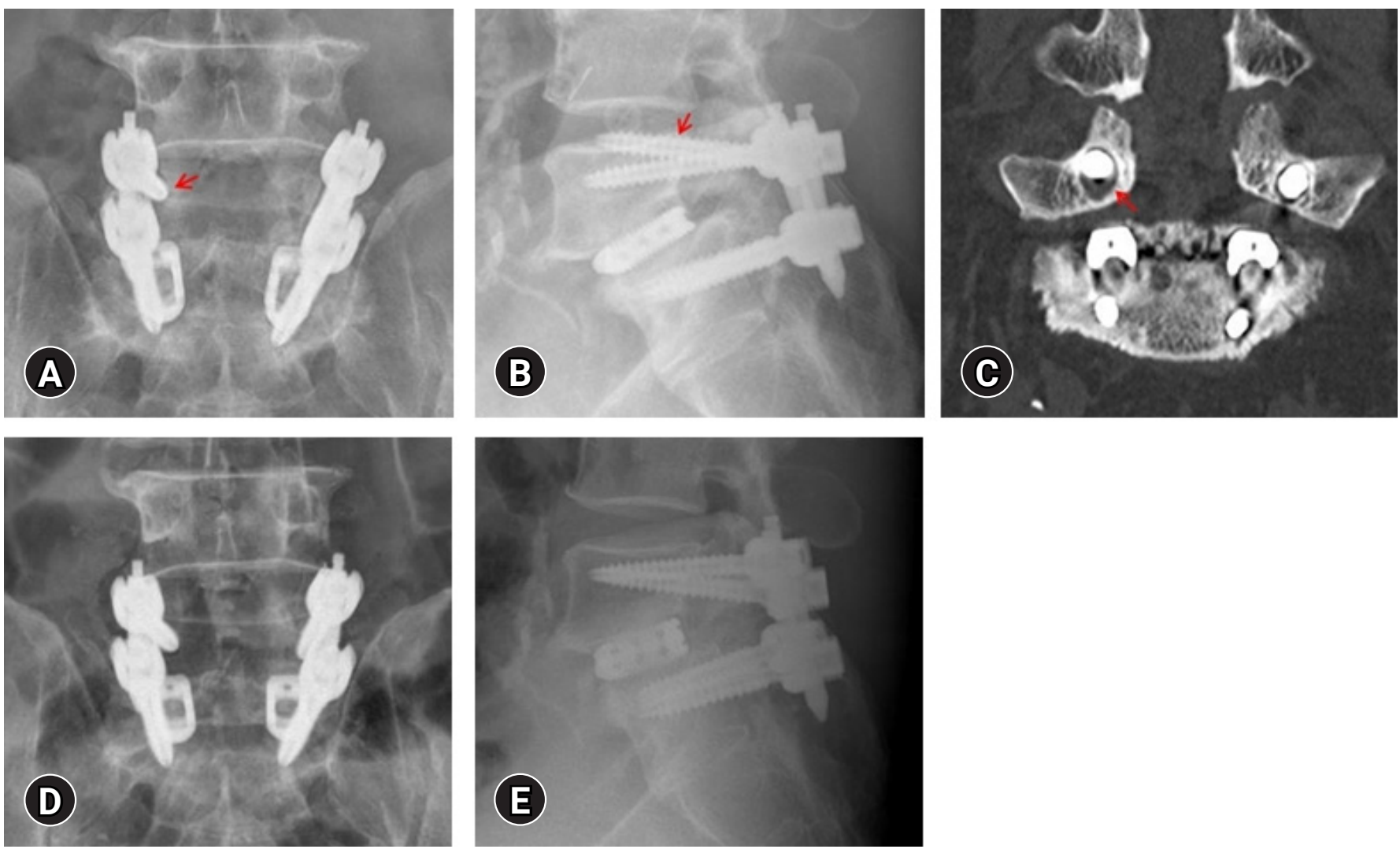

Fig. 3. A 67-year-old male who underwent L5-S1 lumbar interbody fusion 8 months earlier. (A, B) Simple anteroposterior and lateral radiographs show a radiolucent halo around the right L5 pedicle screw (arrow). (C) Coronal computed tomography reveals loosening of the right L5 pedicle screw (arrow). (D, E) Postoperative simple anteroposterior and lateral radiographs obtained 6 months after revision surgery show adequate repositioning of the screws.

(90\%) showed excellent or good outcomes based on the modified MacNab's criteria. Before revision surgery, the mean pain score on VAS was 6.9, which decreased to 3.1 at the final follow-up $(\mathrm{P}<0.05)$. No loosened or stripped screws were observed in any study participant, and no patient required extensive open surgery.

\section{Discussion}

Pedicle screw fixation is an essential technique for spinal fusion surgery. It is widely used, because it provides immediate and strong fixation. However, it often results in complications and symptomatic screw loosening is one of these complications. Pedicle screw loosening is the main cause of morbidity in patients who undergo spinal instrumentation surgery. It is related to altered biome chanics and bone fragility [2].

To avoid screw loosening or implant failure, several options have been proposed, including adding cement augmentation and variously designed screws such as, expandable screws, bicortical screws, and cannulated and fenestrated screws [8]. However, when screw loosening or implant failure occurs, few treatment options exist. The typical treatment involves extensive surgery to remove the screws with extension of instrumentation and reinsertion of more pedicle screws adding cage replacement with the addition of allograft or other bone substitutes [9]. However, all these techniques carry perioperative risks and morbidities and moreover, revision surgery has an expensive cost burden.

Extensive open revision surgery is usually particularly contraindicated for elderly and fragile patients with medical comorbidities. Minimally invasive alternative treatment options using cement augmentation have recently been reported $[5,6]$. They applied a vertebral augmentation technique for pedicle screw loosening to activate screw oversizing and reduce screw micromobility. Cianfoni et al. [10] also reported percutaneous cement augmentation for the treatment of clinically relevant screw loosening, both in avoidance of extensive open surgery and for pain relief without neurologic complications or clinically significant cement leaks.

However, bone resorption around implants can be caused by infections. In cases of clinical and radiologic suspicion of spinal infection, cement augmentation may be contraindicated. The suboptimal sensitivity of spine biopsies and cultures for low-grade infec- 
tions remains a challenge in such cases [11]. Moreover, bone cement augmentation has exothermic properties and leads to frequent complications, such as cement leakage, although most cases are of minor leakages without consequent neurologic deficits.

Xie et al. [12] reported that augmentation of the pedicle screw with both PMMA and autogenous iliac bone transplant in the treatment of degenerative lumbar scoliosis combined with osteoporosis can achieve good surgical results. No significant differences were found between the autogenous bone chip group and the PMMA group with regard to all aforementioned targets, except for the duration of intake of oral pain medication and cost of surgery. Two patients showed asymptomatic cement leakage during surgery in the cement augmentation group. However, harvesting of the autogenous iliac bone graft can be associated with significant morbidity. Minor complications include persistent pain, superficial seromas, and hematomas. Major complications include herniation of abdominal contents, vascular injury, neurologic injuries, and deep infections at the donor site. Moreover, considering that most patients with screw loosening are in the elderly age-group, autogenous bone harvesting can be particularly traumatic. Considering these points, bone allografts may be an alternative to autogenous iliac bone as the trajectory reinforcement material.

Some researchers have suggested using bone grafts in the screw trajectory to increase the strength of the pedicle screw. However, evidence is limited for improvement in the strength of the pedicle screw with use of bone grafts in the screw trajectory. Pfeifer et al. [13] used a milled femoral head allograft bone and a matchstick-shaped bone for trajectory revision and found that using bone grafts in the screw trajectory does not improve the strength of the screw in the failed trajectory. Chrea et al. [14] also utilized allograft bone plugs for trajectory augmentation and found that pullout loads of an augmented screw were not significantly higher than those of an unaugmented screw.

However, recently, Jia et al. [15] reported that augmentation of the trajectory allograft bone using small-diameter cancellous bone particles via a delivery tube before screw insertion can significantly increase the pullout strength and allowed good distribution of the bone at the screw-bone interface. To improve the bone grafting technique, we used a small allograft of cancellous bone particles and changed the screw trajectory using larger-diameter screws.

In the literature, pedicle screw loosening has been associated with decreased pullout strength and extraction torque. It can be regarded as a type of instrument failure and is related to back pain and pseudoarthrosis [16].

Therefore, to prevent repeated pedicle screw loosening, we recommend meticulous screw insertion via a new trajectory, using a longer and thicker screw as well as augmentation of allograft bone particles to prevent the toggling effect. In addition, strict care of the spine with osteoporosis or osteopenia is essential.

In our study, we performed removal of the loosened screws, checked the screw channel, and reimplanted larger screws via a new trajectory filled with allograft bone chips. Our technique has the advantage of being a minimally invasive procedure rather than an extensive open surgery, which is conventionally used for treating symptomatic screw loosening. It can also be applied in patients with low-grade local infection, which is a contraindication for cement injection. In the postoperative control at a minimum of 6 months after surgery and at the final follow-up, none of the screws showed significant loosening, and significant pain reduction was maintained.

However, there are some limitations to the current study. The main limitations are the retrospective study design, small sample size short term follow-up, and an intrinsically subjective definition of instrumentation failure. Furthermore, the subjective nature of self-assessment of pain may be another limitation of this study.

Second, we focused on the screw loosening and did not evaluate the interbody fusion status with the status of implanted cages and disc space. A CT scan was not performed after surgery, we are not able to confirm the correct fusion status. It is necessary to perform a CT scan be at least two years after surgery to confirm the fusion status. Therefore, larger, prospective cohort analyses with longterm follow-ups focused on bone fusion status, and biomechanical studies are required to provide more generalized outcome data and to evaluate the long-term results in terms of bone fusion. Moreover, for patients with pronounced, multilevel screw loosening, extensive open revision surgery should be considered. It is of course important to control osteoporosis during the follow-up period.

The current study suggests that reimplantation of pedicle screws via a different trajectory using a larger-diameter screw for selected low-grade screw loosening can provide a well-tolerated alternative to extensive open surgery for select patients.

\section{Conflicts of interest}

No potential conflict of interest relevant to this article was reported.

\section{ORCID}

Kyoung Hwan Yoo, https://orcid.org/0000-0001-8047-887X

Pius Kim, https://orcid.org/0000-0001-5514-9257

Chang Il Ju, https: / / orcid.org/0000-0001-9123-2808

Seok Won Kim, https://orcid.org/0000-0002-1910-0242 


\section{REFERENCES}

1. Kang SH, Kim KT, Park SW, Kim YB. A case of pedicle screw loosening treated by modified transpedicular screw augmentation with polymethylmethacrylate. J Korean Neurosurg Soc 2011;49:75-8.

2. Pearson HB, Dobbs CJ, Grantham E, Niebur GL, Chappuis JL, Boerckel JD. Intraoperative biomechanics of lumbar pedicle screw loosening following successful arthrodesis. J Orthop Res 2017;35:2673-81.

3. Galbusera F, Volkheimer D, Reitmaier S, Berger-Roscher N, Kienle A, Wilke HJ. Pedicle screw loosening: a clinically relevant complication? Eur Spine J 2015;24:1005-16.

4. Wu ZX, Gong FT, Liu L, et al. A comparative study on screw loosening in osteoporotic lumbar spine fusion between expandable and conventional pedicle screws. Arch Orthop Trauma Surg 2012;132:471-6.

5. Amoretti N, Bertrand AS, Gallo G, et al. Percutaneous consolidation of loosened spine arthrodesis under CT and fluoroscopy guidance by radiologists: a new useful technique. Eur Radiol 2015;25:1135-9.

6. Fu TS, Li YD. Fluoroscopy-guided percutaneous vertebroplasty for symptomatic loosened pedicle screw and instrumentation-associated vertebral fracture: an evaluation of initial experiences and technical note. J Neurosurg Spine 2018;28:364-71.

7. Zimmerli W. Clinical practice. Vertebral osteomyelitis. N Engl J Med 2010;362:1022-9.

8. Fransen P. Increasing pedicle screw anchoring in the osteoporotic spine by cement injection through the implant: technical note and report of three cases. J Neurosurg Spine 2007;7:366-9.

9. McAfee PC, Weiland DJ, Carlow JJ. Survivorship analysis of pedicle spinal instrumentation. Spine (Phila Pa 1976) 1991; 16(8 Suppl):S422-7.

10. Cianfoni A, Giamundo M, Pileggi M, et al. Spinal instrumentation rescue with cement augmentation. AJNR Am J Neuroradiol 2018;39:1957-62.

11. Prodi E, Grassi R, Iacobellis F, Cianfoni A. Imaging in spondylodiskitis. Magn Reson Imaging Clin N Am 2016;24:581-600.

12. Xie Y, Fu Q, Chen ZQ, et al. Comparison between two pedicle screw augmentation instrumentations in adult degenerative scoliosis with osteoporosis. BMC Musculoskelet Disord 2011; 12:286.

13. Pfeifer BA, Krag MH, Johnson C. Repair of failed transpedicle screw fixation: a biomechanical study comparing polymethylmethacrylate, milled bone, and matchstick bone reconstruction. Spine (Phila Pa 1976) 1994;19:350-3.

14. Chrea B, Malempati H, Campbell JR, Khan S, Ching RP, Lee MJ. Enhancing pedicle screw fixation in the lumbar spine using allograft bone plug interference fixation. J Spinal Disord Tech 2014;27:E81-7.

15. Jia C, Zhang R, Xing T, et al. Biomechanical properties of pedicle screw fixation augmented with allograft bone particles in osteoporotic vertebrae: different sizes and amounts. Spine J 2019; 19:1443-52

16. Wetzel FT, Brustein M, Phillips FM, Trott S. Hardware failure in an unconstrained lumbar pedicle screw system: a 2-year follow-up study. Spine (Phila Pa 1976) 1999;24:1138-43. 